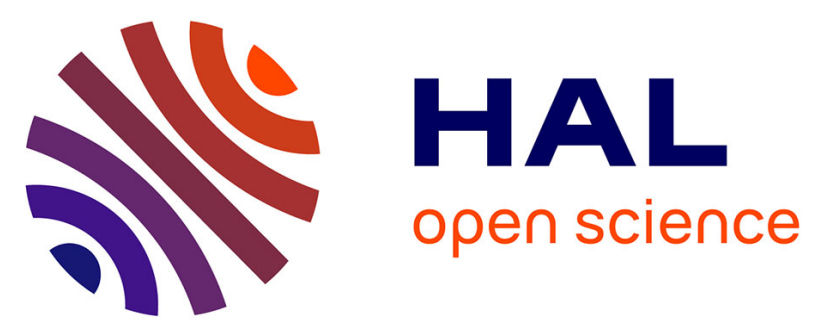

\title{
Picosecond Joule heating in photoconductive switch electrodes
}

\author{
Bjorn Vermeersch, Gilles Pernot, Hong Lu, Je-Hyeong Bahk, Arthur C. \\ Gossard, Ali Shakouri
}

\section{To cite this version:}

Bjorn Vermeersch, Gilles Pernot, Hong Lu, Je-Hyeong Bahk, Arthur C. Gossard, et al.. Picosecond Joule heating in photoconductive switch electrodes. Physical Review B: Condensed Matter and Materials Physics (1998-2015), 2013, 88 (21), pp.214302. 10.1103/PhysRevB.88.214302 . hal-01535974

\section{HAL Id: hal-01535974 \\ https://hal.univ-lorraine.fr/hal-01535974}

Submitted on 9 Jun 2017

HAL is a multi-disciplinary open access archive for the deposit and dissemination of scientific research documents, whether they are published or not. The documents may come from teaching and research institutions in France or abroad, or from public or private research centers.
L'archive ouverte pluridisciplinaire HAL, est destinée au dépôt et à la diffusion de documents scientifiques de niveau recherche, publiés ou non, émanant des établissements d'enseignement et de recherche français ou étrangers, des laboratoires publics ou privés.

\section{(1)(1) $\$(0)$}

Distributed under a Creative Commons Attribution - NonCommercial - ShareAlikel 4.0 


\title{
Picosecond Joule heating in photoconductive switch electrodes
}

\author{
Bjorn Vermeersch, ${ }^{1,2, *}$ Gilles Pernot, ${ }^{2, \dagger}$ Hong Lu, ${ }^{3}$ Je-Hyeong Bahk, ${ }^{1,2}$ Art Gossard, ${ }^{3}$ and Ali Shakouri ${ }^{1,2, \ddagger}$ \\ ${ }^{1}$ Birck Nanotechnology Center, Purdue University, West Lafayette, Indiana 47907, USA \\ ${ }^{2}$ Baskin School of Engineering, University of California, Santa Cruz, California 95064, USA \\ ${ }^{3}$ Materials Department, University of California, Santa Barbara, California 93106, USA
}

(Received 24 July 2012; revised manuscript received 18 October 2013; published 11 December 2013)

\begin{abstract}
We present experimental observations of picosecond Joule heating inside the gold metallization of ErAs:GaAs photoconductive switches. Femtosecond laser time-domain thermoreflectance is employed to resolve the fast thermal dynamics in the central switch electrode during generation and transmission of rf electrical pulses. Sharp features in the thermoreflectance signal scaling quadratically with the bias/pulse voltage reveal Joule heating with durations $\leqslant 5 \mathrm{ps}$ inside the gold metal. The temporal shape and rise time of the signals is in excellent agreement with the theoretical pulse wave form and subpicosecond carrier lifetime of the active medium. Probing different locations on the electrode shows the propagation, attenuation, and dispersion of the electrical pulses along the coplanar waveguide structure. Overall, the presented photothermal experiments demonstrate great potential to reveal the internal dynamics of photoconductive switches, characterize transmission lines, and study ultrafast heating in metals.
\end{abstract}

DOI: 10.1103/PhysRevB.88.214302

PACS number(s): 66.70.Df, 44.10.+i, 72.15.Eb, 78.47.jg

\section{INTRODUCTION}

A photoconductive switch (PCS), also known as an Auston switch after its inventor, ${ }^{1}$ is an integrated semiconductor device whose electrical state can be altered between virtually insulating and highly conductive by means of optical stimuli. The active area consists of a gap in a metal electrode deposited on top of a semi-insulating (SI) semiconductor substrate. Without external stimulation, the device is, in essence, an open circuit (off state). Illumination of the gap at a suitable optical wavelength generates electron-hole pairs in the substrate which enable current to flow from one side of the electrode to the other (on state). A large breakdown field and short carrier lifetime of the photoconductive medium are of particular interest to the device performance. The breakdown field directly determines the electrical bias that can be applied over an electrode gap of a given width. The short lifetime, meanwhile, induces a fast decay of the photoinduced carriers after removal of the optical stimulus and is therefore crucial when fast switching is desired. Short lifetimes can be achieved by purposely introducing crystal defects or scatter centers either during or after the material growth. ${ }^{2}$ Low-temperature grown and erbium-doped gallium arsenide (LT GaAs, Er:GaAs) have commonly been used for PCS applications over several decades. ${ }^{3-5}$ More recent work has demonstrated that the inclusion of selfassembling ErAs nanoparticles into the GaAs matrix offers tunable lifetimes and improves overall performance. ${ }^{6,7}$ Fast switching capabilities and other favorable characteristics have led to photoconductive switches being widely employed in the generation and detection of ultrafast electrical pulses, ${ }^{5}$ pulsed microwave power and radar technologies, ${ }^{8}$ and terahertz (THz) imaging applications. ${ }^{9}$ A limited number of studies were devoted to the power dissipation and thermal behavior of PCSs. ${ }^{10-14}$ These typically focus on high-power devices, where peak powers being switched commonly amount to on the order of several megawatts and therefore pose significant thermal management challenges to the overall system. Steadystate models are available to estimate the heat-sink area required to maintain the semiconductor below a given target temperature. ${ }^{10}$ Transient analyses have studied the power wave form during switching ${ }^{11}$ and thermal runaway failures attributed to current filamentation. ${ }^{12-14}$ Due to their larger electrical and thermal masses, characteristic switch times and thermal time constants of high-power PCSs are on the order of $50 \mathrm{~ns}$ and $1 \mu \mathrm{s}$, respectively. ${ }^{13}$ Microscale, low-power devices will exhibit dynamics that are several orders of magnitude faster. In addition, they offer an interesting potential to study picosecond thermal dynamics in other electronic and thermoelectric integrated devices. ${ }^{15}$

In the present study, however, we focus on the thermal behavior inside a PCS. Namely, we monitor the temperature change of the switch electrode during transmission of picosecond electrical pulses. It is well known that thermal transients can occur very rapidly in metals due to the ultrafast heating of the free electrons ${ }^{16}$ and subsequent fast energy interaction with the lattice phonons. ${ }^{17}$ Theoretical models ${ }^{17-19}$ and experimental verifications ${ }^{19-22}$ have shown that the surface temperature of thin metal films subjected to an energy impulse responds virtually instantaneously and then decays rapidly with time constants well below 1 ps. All of these studies were based on optical stimulation of the metal; that is, the heating of the film is induced by a laser pulse. To the best of our knowledge, studies on picosecond thermal dynamics in metals induced by electrical impulses have not been published.

In this paper, we report ultrafast Joule heating inside a PCS gold electrode induced by the transmission of rf electrical pulses. Femtosecond laser thermoreflectance is used to resolve the thermal transients with subpicosecond resolution. At short pump-probe delays, the thermal signal clearly shows sharp features less than 5 ps (FWHM) wide with magnitudes that depend quadratically on the pulse voltage. From the shape of the photothermal signal we extract a carrier lifetime of $\approx 0.9 \mathrm{ps}$, in excellent agreement with earlier independent measurements. Secondary temperature peaks that can be attributed to electrical pulse reflections from the wire bonds and package are also observed. Finally, by monitoring the temperature 

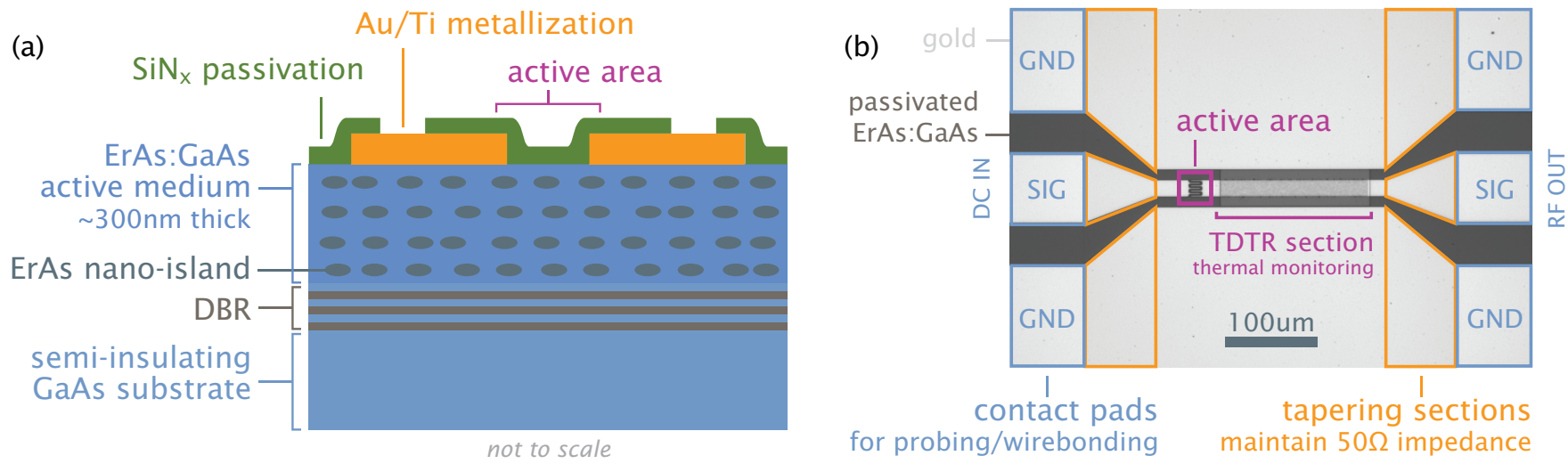

(c)

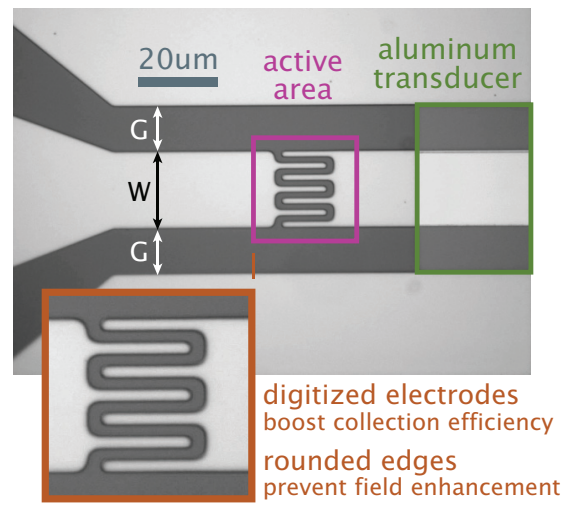

(d)
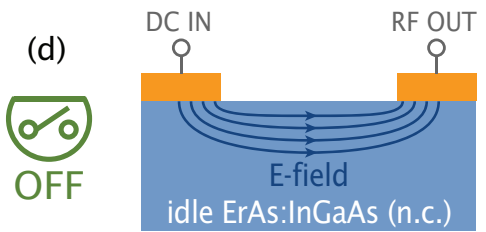

DC IN laser beam RF OUT

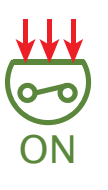

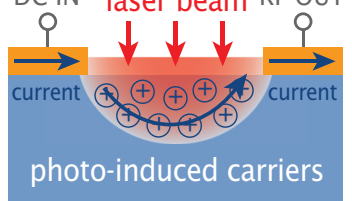

(e)
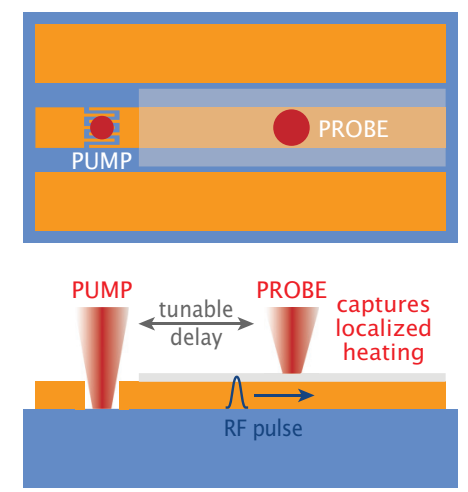

FIG. 1. (Color online) Fabricated photoconductive switch samples: (a) cross-section structure, (b) micrograph of a typical device, (c) detailed view of the active area, (d) schematic switching principle, and (e) schematic illustration of electrical and thermal processes during measurement.

change at different locations on the electrode, we can reveal the electrical pulse propagation and attenuation along the coplanar waveguide inside the device. The experimental wave propagation speed, $39 \%$ of the speed of light, closely matches textbook predictions.

\section{SAMPLE DESIGN AND FABRICATION}

We have fabricated photoconductive switches based on ErAs:GaAs grown by molecular beam epitaxy (MBE) as an active medium. The schematic cross-section structure of the samples is shown in Fig. 1(a). We have chosen a so-called optical cavity layout. ${ }^{23,24}$ The active layer has a thickness on the order of the optical penetration length and lies on top of a distributed Bragg reflector (DBR). The resulting concentration of light at the upper regions of the photoconductive film, where the electric field and photoconductive gain are highest, ${ }^{23}$ improves the quantum efficiency of the devices. ${ }^{23,24}$ Starting from a SI GaAs substrate, we first deposit an AlAs/AlGaAs superlattice to form the DBR and then proceed with growing the active layer. During the latter, the Ga and As supplies are turned off at periodic intervals and are replaced by Er injection. Driven by surface chemistry, the Er atoms self-assemble with the remaining As background pressure in the chamber into $\approx 1-2-n m$ ErAs nanoislands. ${ }^{6}$ The growth process results in a periodic stack of parallel planes of $\approx 1.2$ monolayers of ErAs embedded in the GaAs matrix. The ErAs islands introduce energy levels inside the GaAs band gap. They therefore act as efficient trap centers that can recapture the photoinduced carriers far more quickly than intrinsic thermal recombination. ${ }^{6}$ Moreover, the resulting carrier lifetime is tunable by changing the spacing $L$ between the ErAs planes. ${ }^{7}$ Smaller spacings induce shorter lifetimes at the expense of increased dark (leakage) currents. ${ }^{7}$ Our samples feature $L \approx 60 \mathrm{~nm}$, inducing a typical lifetime slightly below 1 ps. $^{7}$ The surface metallization of the medium is carried out using $\approx 300 \mathrm{~nm}$ of e-beam evaporated gold on a 20-nm titanium adhesion layer. The gold thickness is chosen as a compromise between minimizing the thermal mass of the metal and still maintaining good electrical performance. The measured electrical conductivity of the electrodes was $\sigma=3.1 \times 10^{7} \mathrm{~S} / \mathrm{m}$, roughly $70 \%$ of typical Au bulk values. We note here that the associated skin depth $\delta \approx \sqrt{\frac{2}{\sigma \omega \mu_{0}}}$ still amounts to $\approx 225 \mathrm{~nm}$ for $\omega=10^{12} \mathrm{rad} / \mathrm{s}$. This means the entire electrode thickness is utilized for electromagnetic transport, even for picosecond pulses. The lift-off photoresist method is used to pattern the metal layer and define the actual devices. The lithography is carried out on a mask aligner and wafer stepper using 5:1 projection. The technique provides features with clean edges and 200-250-nm lateral resolution at the wafer plane. Finally, the sample is passivated with $\mathrm{SiN}_{x}$ by plasma-enhanced chemical vapor deposition (PECVD) for surface protection and optical optimization. The nitride layer is transparent to the laser wavelength of $785 \mathrm{~nm}$ and works, at the chosen thickness of $\approx 300 \mathrm{~nm}$, as an antireflection coating to maximize the photon influx on the active area. The electrode sections that will be thermally monitored are left unpassivated and then are subsequently covered by an evaporated aluminum transducer for the thermoreflectance measurements (see the 
next section). The transducer has a thickness of $53 \mathrm{~nm}$, as determined from picosecond ultrasonics.

We designed a variety of switches, with active areas ranging from $10 \times 10 \mu \mathrm{m}^{2}$ to $20 \times 20 \mu \mathrm{m}^{2}$. Figures 1 (b) and 1(c) show a top-view micrograph of a typical device and a detail of its active area. Transmission line metallization is required to carry the generated high-speed electrical pulses. We have opted for a coplanar waveguide (CPW) structure, i.e., a signal-carrying electrode (width $W$ ) centered in between two surrounding ground planes (separation gap $G$ ). This layout provides full single-sided access to the samples from the top, which is convenient for probing and wire bonding. Moreover, the characteristic CPW impedance is predominantly determined by the ratio of the ground gap to the electrode width ${ }^{25} G / W$. This makes it possible to use tapering structures between the contact pads and actual devices without sacrificing impedance matching [see Fig. 1(b)]. Empirical correlations ${ }^{25}$ evaluated for 300-nm gold CPWs with $70 \%$ bulk conductivity on a $500-\mu \mathrm{m} \mathrm{GaAs}$ substrate, determined that $G / W=0.6$ provides the desired impedance $Z_{0}=50 \Omega$. Time-domain voltage reflectometry measurements with a high-speed sampling scope on a test line several millimeters in length showed a nearly perfect match to $50 \Omega$. Finally, we remark that the aluminum transducers deposited on the central electrodes turned out to be significantly wider than intended [Figs. 1(b) and 1(c)]. This artifact is possibly due to a faulty lithography exposure of the corresponding mask layer. However, thanks to the passivation layer separating them electrically and thermally from the wafer material underneath, these aluminum overhangs were found not to be detrimental to the device operation or thermal characterization.

To maximize the device performance and reliability, specific care is given to the design of the photoconductive gaps (active switch area). We can expect that electrodes with multiple fingers stretching into the gap offer a trade-off between improved carrier collection efficiency and reduced optical fluence. We found that such interdigited structures produced pulse amplitudes up to twice as large as simple gap geometries. Figure 1(c) shows a close-up of a device with central electrode width $W=19.5 \mu \mathrm{m}$ with fingers and interdigit spacing both $1.5 \mu \mathrm{m}$ wide. This geometry provided the largest pulse output of all available designs, and this sample was used for each of the measurements described further. Biases up to $15 \mathrm{~V}$ are supplied, corresponding to effective fields of $10^{7} \mathrm{~V} / \mathrm{m}$, or roughly one quarter of the breakdown field of high-purity GaAs. ${ }^{26}$ We therefore included rounded edges near all active areas in the metallization mask in an attempt to prevent field-enhancement effects.

After fabrication the wafer was diced into separate chips which were then mounted in a metal package. Wire bonds connect the on-chip contact pads to gold-on-Kapton strips glued to the baseplate with epoxy on either side of the chip. The strips then finally connect to two SubMiniature version A (SMA) connectors embedded in the package (one for dc bias input and the other for the rf pulse output). Physical processes during the measurement are schematically illustrated in Figs. 1(d) and 1(e).

\section{EXPERIMENTAL METHODS}

We utilize a time-domain thermoreflectance (TDTR) system with double-sided modulation scheme (Fig. 2).

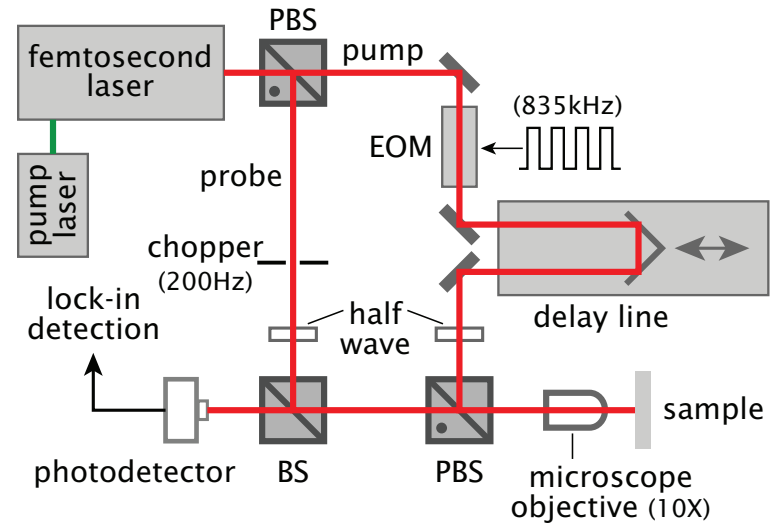

FIG. 2. (Color online) Femtosecond laser system for pump-probe thermoreflectance measurements. EOM and (P)BS indicate electrooptic modulator and (polarizing) beam splitter, respectively.

Pulses from a $Q$-switched Ti:sapphire laser (central wavelength of $785 \mathrm{~nm}$, pulse width $\approx 250 \mathrm{fs}$, pulse repetition of $75.8 \mathrm{MHz}, 700 \mathrm{~mW}$ at cavity output) are split into a pump and probe beam which are then recombined and focused onto the sample with a $10 \times$ microscope objective lens. The $1 / e^{2}$ pump and probe beam radii at the focal plane are $6.5 \pm 0.5$ and $9 \pm 0.6 \mu \mathrm{m}$, respectively, as measured with a beam profiler (knife-edge slit method). We typically set the pump and probe powers of the sample to 40 and $8 \mathrm{~mW}$, respectively. The pump beam is square wave modulated at $835 \mathrm{kHz}$ with an electro-optic modulator (EOM) and is positioned over the active area of the biased photoconductive switch to generate rf electrical pulses. The pump path also comprises a mechanical delay line that enables us to adjust the relative arrival time of pump and probe beams on the sample with subpicosecond resolution. We used a two-photon detector to accurately determine the incidence point (temporal overlap of pump and probe). The probe beam is mechanically chopped at $200 \mathrm{~Hz}$ and monitors the reflectivity of the PCS electrode. The rf lock-in detection at $835 \mathrm{kHz}$ of the detector signal and subsequent demodulation at $200 \mathrm{~Hz}$ provides the thermally induced reflectivity variations of the electrode. The double-modulation scheme rejects the influence of residual pump reflections. The signal-to-noise ratio (SNR) is further improved by a pure inductance filter between the detector and rf lock-in. ${ }^{27}$ The inductor forms a resonant circuit with the detector capacitance that amplifies signals in the $835-\mathrm{kHz}$ range by about an order of magnitude while suppressing all other modulation harmonics. Further details about laser thermoreflectometry can be found elsewhere. ${ }^{28} \mathrm{We}$ recall that a thin aluminum film was deposited on top of the sample electrodes. This is because aluminum shows a relatively large change in reflectivity with temperature under near-IR illumination, making it a favorable transducer material for these experiments. ${ }^{29}$ The thermoreflectance coefficient of gold peaks in the blue and green portions of the visible spectrum but then drops quickly towards longer wavelengths. ${ }^{30}$ We were nevertheless able to obtain a signal from a bare gold electrode as well, in accordance with published data on optically pumped transducerless gold structures. ${ }^{19,21}$

We use continuous acquisition in our system; that is, the mechanical delay line moves uninterruptedly at a constant 

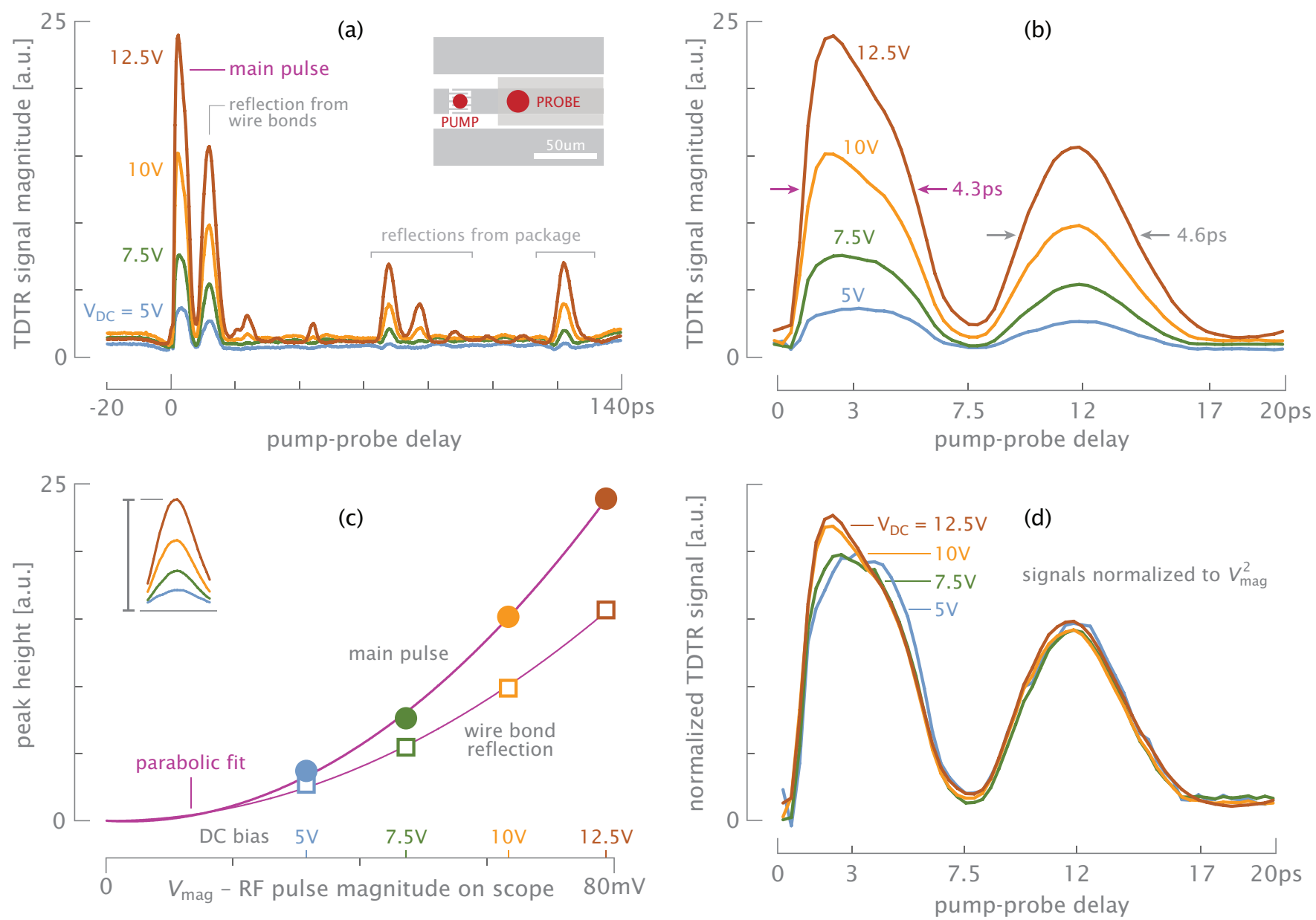

FIG. 3. (Color online) Influence of electrical bias on thermoreflectance signal obtained in fixed electrode location close to active area: (a) high-resolution scan of moderate pump-probe delays, (b) detailed view of initial transients, (c) signal peak heights vs rf pulse magnitude $V_{\text {mag }}$, and (d) signals normalized to $V_{\text {mag }}^{2}$.

speed to take data over a pump-probe delay range. Multiple scanning sweeps are averaged to boost the SNR. By limiting the translation speed to $70 \mu \mathrm{m} / \mathrm{s}$, the line moves about $50 \mu \mathrm{m}$ during the settling time of both lock-in amplifiers combined $(\approx 700 \mathrm{~ms})$. This gives an effective temporal resolution of 330 fs for the measurement. Attempting to move the line even slower would not necessarily resolve even faster thermal dynamics as the responses are convoluted with the Gaussian shape $(\geqslant 250$ fs FWHM) of the laser pulses anyway.

During the measurements, a dc voltage supply provides the bias to one side of the switch, while the other side is connected to a 3.5-GHz scope. The latter allows us to monitor the electrical pulse output while also acting as a $50 \Omega$ load termination. While the scope has a bandwidth that is highly insufficient to resolve the rf pulse dynamics (channel rise time $\approx 130 \mathrm{ps}$ ), it still offers an accurate relative measure of the pulse magnitude (conservation of electric energy).

The main advantage of using TDTR experiments is their ability to observe ultrafast thermal transitions induced by the rf electrical pulses, rather than the voltage wave form itself. The method offers subpicosecond temporal resolution but without any need for $\mathrm{GHz} / \mathrm{THz}$ bandwidth electronics. In addition, the pulse transmission can be easily tracked along the electrode by simply positioning the probe beam on any desired location.
Electrical observations are more invasive and less flexible, as they would require physical probing pads that need to be incorporated beforehand into the sample design.

\section{RESULTS AND DISCUSSION}

Prior to the TDTR experiments, we characterized the influence of the dc bias (2.5-12.5 V) and optical pump power $(5-40 \mathrm{~mW})$ on the rf pulse output to verify proper working of the device. The pulse magnitude scales linearly with dc bias, while it varies with pump power as a power law with an exponent of 0.33 . The substantially sublinear dependence on the optical stimulus (or equivalently, the carrier density in the active area) has also been observed in other studies ${ }^{7}$ and can be attributed to screening of the external bias field ${ }^{31}$ and nonlinear terms in the recombination equations. ${ }^{32}$

For the first set of thermoreflectance measurements, we position the probe beam on the central PCS electrode close to the active area and study the TDTR signal as a function of the pump-probe delay for a variety of bias voltages. The results are summarized in Fig. 3. A number of sharp features are clearly visible at short delays [Fig. 3(a)], suggesting the presence of thermal transients occurring over time windows of $10 \mathrm{ps}$ and less. The peaks become increasingly more pronounced 
with growing bias voltage. Otherwise, the rest of the signal features, i.e., the peak locations and overall shape, remain essentially unchanged. As we will demonstrate further, the most prominent peak, immediately after the optical pump event, is related to the main electrical rf pulse. The second, smaller peak and subsequent peak clusters at intermediate pump-probe delays can be attributed to electrical pulse reflections from, respectively, the wire bonds and package. It is interesting to point out that in between the peaks, the signal fully returns to the baseline. For most structures commonly studied with TDTR, namely, a transducer on a dielectric thin film or substrate, the laser repetition rate (13.2 ns in our system) is far too short for the surface temperature to fully decay back to the ambient level before the next pulse arrives. The resulting pulse accumulation effects cause the TDTR signal to deviate significantly from the single pulse response of the sample. ${ }^{33}$ The fast transitions in our results indicate that the electrode rapidly returns to thermal equilibrium, in accordance with the ultrafast electron and phonon processes inside the gold metal. A closer inspection of the initial transients near zero pump-probe delays, shown in Fig. 3(b), reveals dynamics with FWHM pulse widths of 4.3 and 4.6 ps for the main and secondary peaks, respectively.

To quantify the influence of the electrical bias on the thermal signal, we plot the height of the TDTR peaks as a function of the magnitude of the electrical pulse output observed on the scope. The data closely follow a parabolic trend [Fig. 3(c)], suggesting that peak height is proportional to $V_{\text {mag }}^{2}$. In double-logarithmic scale, the peak heights closely follow straight lines, with slopes of 2.06 and 1.92 for the first and second peaks, respectively. This is again indicative of a quadratic relation (power law with an exponent of $\approx 2$ ) between TDTR signal strength and electrical bias. These analyses thus strongly suggest the thermoreflectance measurements are observations of picosecond Joule heating in the electrode induced by the transmitted electrical pulses. A voltage pulse $V_{p}(t)$ propagating on the coplanar waveguide is indeed associated with a power dissipation $V_{p}^{2}(t) / Z_{0}=I_{p}(t)^{2} Z_{0}$, which will induce a proportional temperature rise. In the absence of electrical bias $\left(V_{d c}=0\right)$ the signal reduced to a single peak that decays sharply with a time constant below $1 \mathrm{ps}$ and with peak magnitude roughly 50 times weaker compared to the $V_{d c}=12.5 \mathrm{~V}$ data. These optically induced dynamics have, as mentioned earlier, been studied in detail by other authors. ${ }^{17-22}$ Under electrical bias, the TDTR signals become very similar after normalization to the square of the pulse magnitude $V_{\text {mag }}^{2}$, although we observe a small difference in the main peak between lower and higher biases [Fig. 3(d)]. We partly attribute the discrepancy to small drifts in the spatial alignment of the sample and laser beams, as confirmed further. The data were taken sequentially over the course of about $1.5 \mathrm{~h}$ (20 min of averaging four scan sweeps for each curve). In addition, it is not unlikely that the increasing bias also alters the actual physical switching behavior. Earlier studies have shown that the occurrence of double-injection effects and current filamentation appearing under high-field conditions directly influence the shape of the electrical pulses. ${ }^{12}$

Accounting for the near-instantaneous thermal response of gold cited earlier and the subpicosecond width of the laser pulses, we can estimate the electrical pulse duration (FWHM)

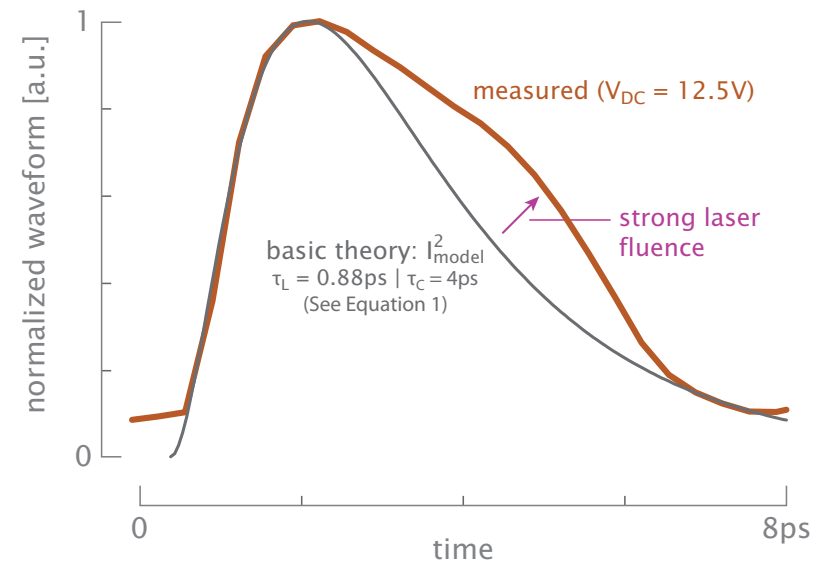

FIG. 4. (Color online) Comparison of the main peak in the TDTR signal to the theoretical pulse power wave form.

to be 3-4 ps. In a first-order approximation, the current pulse generated by a PCS can be described as ${ }^{2,34}$

$$
I_{\text {model }}(t) \propto\left|\exp \left(-t / \tau_{L}\right)-\exp \left(-t / \tau_{C}\right)\right|,
$$

where $\tau_{L}$ is the carrier lifetime of the switch medium and $\tau_{C}$ is a capacitive time constant governing the turnoff characteristics. The resulting power wave form $\propto I_{\text {model }}^{2}(t)$ can be fitted reasonably well to the TDTR data when setting $\tau_{L}=0.88 \mathrm{ps}$ and $\tau_{C}=4$ ps (Fig. 4).

The obtained lifetime is in excellent agreement with the value $\lesssim 1$ ps determined earlier in an independent study from direct optical probing of the photoconductive medium. ${ }^{7} \mathrm{We}$ note that a time delay of $0.35 \mathrm{ps}$ is applied to the model to match the measurements. We can attribute this horizontal offset in the experiments to the electrical propagation time between the active area and probe location [delay $\approx 47 \mu \mathrm{m} /(0.39 c) \approx$ $0.4 \mathrm{ps}$; see further details below] and a small error on the pumpprobe incidence point. The convolution of the theoretical curve with the Gaussian temporal shape of the laser pulses was verified to have negligible effects on the wave form appearance and is therefore omitted for simplicity. We notice an anomaly during the turnoff; namely, the experimental curve is clearly concave up rather than concave down as predicted by the theory. This transition in the electrical pulse shape has been observed in other studies ${ }^{35}$ and is provoked by large optical fluences and electric fields. The mechanism is likely related to GaAs intervalley scattering and thermal generation of carriers during self-heating inside the active switching area. ${ }^{35}$

Our second set of measurements consists of probing four different locations along the PCS electrode under a constant bias of $12.5 \mathrm{~V}$ (Fig. 5). The fast thermal response of the gold, the strong spatial decay for lateral heat spreading, and the virtual absence of accumulation effects at short pumpprobe delays allows us to consider the different locations on the electrode as thermally independent. Overall, the signals are very similar for each position, although some differences are visible in the sharp features [Fig. 5(a)]. A closer inspection of the main peak reveals that as we move farther away from the active area, the signal starts later and becomes weaker [Fig. 5(b)]. We can attribute these effects to the wave propagation and attenuation of the electrical pulses, 

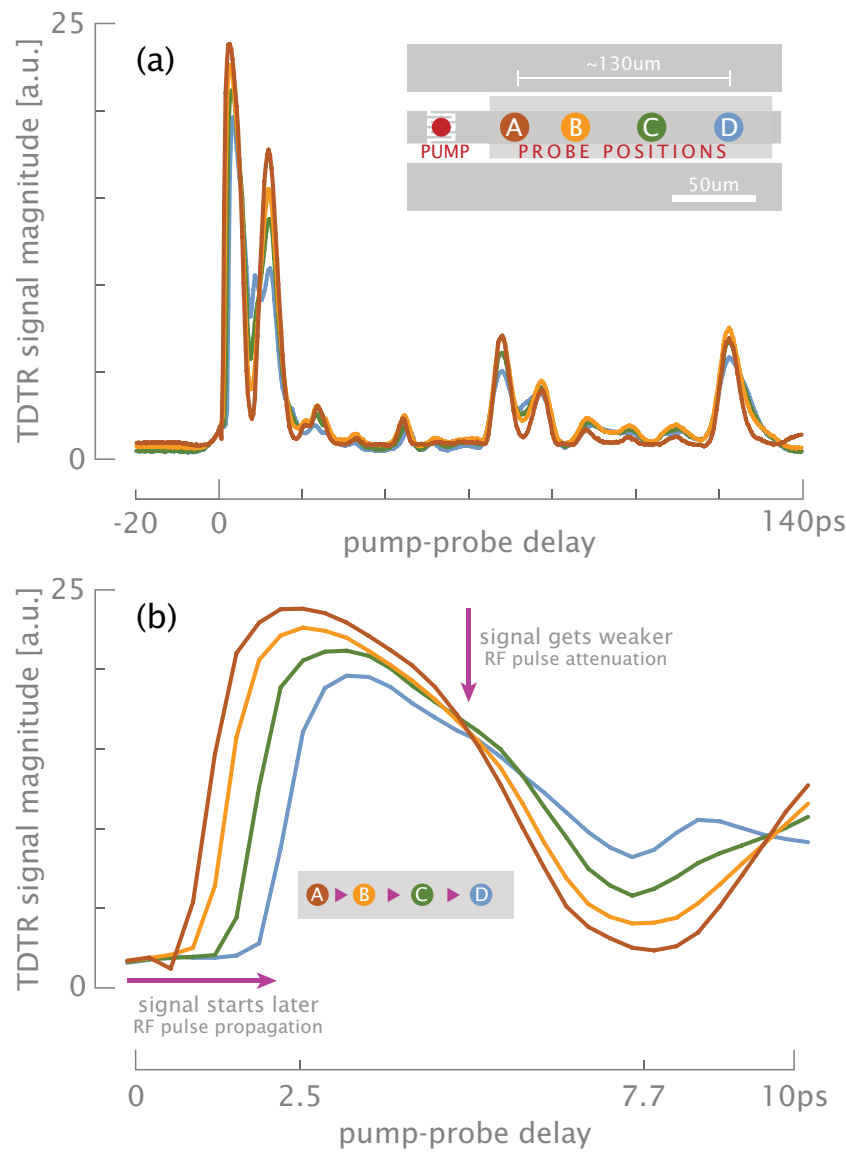

FIG. 5. (Color online) Influence of electrode position on thermoreflectance signal obtained under fixed electrical bias: (a) highresolution scan of moderate pump-probe delays and (b) detailed view of initial transients.

respectively. We found that the rising edges of normalized signals for positions A and D, roughly $130 \mu \mathrm{m}$ apart, aligned perfectly when a time shift of $1.1 \mathrm{ps}$ was applied. The inferred wave propagation speed of about $118 \mu \mathrm{m} / \mathrm{ps}$, i.e., $39 \%$ of the speed of light $(0.39 c)$, deviates less than $5.5 \%$ from theoretical predictions. Textbook correlations ${ }^{25}$ for the fabricated gold coplanar waveguide on GaAs $\left(\epsilon_{r}=12.9\right)$ give an effective dielectric constant of $\epsilon_{r \text {,eff }}=7.37$, i.e., a predicted speed $c / \sqrt{7.37} \approx 0.37 c$. The pulse attenuation is harder to quantify accurately due to alignment uncertainties between the measurements. Relative movements of either pump (changes rf pulse output) or probe (changes optical signal on the detector) will notably alter the absolute magnitude of the signal. Still, the power loss estimated from the decay between positions $A$ and $\mathrm{D}[\approx 10 \log (26 / 21.4) \mathrm{dB} / 130 \mu \mathrm{m} \approx 6500 \mathrm{~dB} / \mathrm{m}]$ is on the same order of magnitude as theoretical predictions $(\approx 2200 \mathrm{~dB} / \mathrm{m}$ at $1 \mathrm{THz})$.

Having determined the pulse propagation speed, we can trace the origin of the secondary peaks in the TDTR measurements to electric pulse reflections. For position A, the second peak appears roughly $7.5 \mathrm{ps}$ after the first one [Fig. 3(b)]. The corresponding one-way travel of about $440 \mu \mathrm{m}$ matches well with the location where the wire bonds leave the contact pads on the rf side and become suspended in the air. We can expect this causes a change in characteristic impedance with associated partial pulse reflection. The fact that the second peak develops progressively earlier as we move farther down the electrode [Fig. 5(b)] supports this viewpoint. In a similar fashion we can attribute subsequent peaks to reflections from the other end of the wire bonds and structures on the package. Note that the reflected pulses become systematically wider and have a more rounded, symmetric shape. The second peak, for example, has slightly widened from 4.3 to 4.6 ps and lacks the lifetime-induced sharp edge present in the first peak [Fig. 3(b)]. This indicates dispersion and pulse broadening in the coplanar waveguide and wire bonds. The effects are exacerbated by the packaging. Measurements of the electrical rf output with a high-speed sampling scope showed a near-Gaussian pulse profile with 45-ps FWHM, one order of magnitude wider than the initial wave form. A similar external pulse width was inferred from autocorrelation experiments with overlapping pump and probe beams on the active area. This technique exploits the nonlinear dependence of the rf pulse output on optical fluence, inducing a dip in the dc average output current.

\section{CONCLUSIONS}

In summary, we have measured picosecond Joule heating in gold. We employ time-resolved laser thermoreflectance to monitor photoconductive switch electrodes during generation and transmission of rf electrical pulses. At short pump-probe times, the signals reveal sharp transients with durations on the order of 4 ps and peak magnitudes that vary quadratically with the pulse/bias voltage. A good match between the signal shape and theoretical power pulse wave form further confirms a direct correlation to the high-speed electrical dissipation in the electrode. The fitted carrier lifetime of $0.88 \mathrm{ps}$ is in excellent agreement with earlier detailed characterizations of the ErAs:GaAs photoconductive medium. Measurements at different locations on the electrode revealed the propagation, attenuation, and dispersion of the electric pulses along the coplanar waveguide. Realistic values are obtained for the transmission velocity (39\% of the speed of light) and power attenuation $(6500 \mathrm{~dB} / \mathrm{m})$. Overall, the presented photothermal experiments are capable of providing valuable insight into internal dynamics of photoconductive switches and ultrafast heat transport in metals, as well as characterizing integrated transmission lines without the overhead of sliding contact or gating structures.

\section{ACKNOWLEDGMENTS}

B.V. thanks Forest Martinez-McKinney at the Santa Cruz Institute for Particle Physics (SCIPP) for his invaluable assistance in wire bonding the samples. A.S. acknowledges financial support from AFOSR/MURI Grant No. FA9550-081-0340 under a subcontract from the University of Michigan. A.S., H.L., and A.G. acknowledge financial support from the Center for Energy Efficient Materials (CEEM), an Energy Frontier Research Center (EFRC) funded at UCSB by the DOE Office of Basic Energy Sciences for material development and fabrication. 
"bvermeer@purdue.edu

${ }^{\dagger}$ Present address: LOMA, Université Bordeaux 1, 33405 Talence, France.

${ }^{\ddagger}$ shakouri@purdue.edu

${ }^{1}$ D. H. Auston, Appl. Phys. Lett. 26, 101 (1975).

${ }^{2}$ D. H. Auston, IEEE J. Quantum Electron. 19, 639 (1983).

${ }^{3}$ S. Gupta, S. Sethi, and P. K. Bhattacharya, Appl. Phys. Lett. 62, 1128 (1993).

${ }^{4}$ S. Sethi, T. Brock, P. K. Bhattacharya, J. Kim, S. Williamson,

D. Craig, and J. Nees, IEEE Electron Device Lett. 16, 106 (1995).

${ }^{5}$ M. Bieler, K. Pierz, and U. Siegner, Appl. Phys. Lett. 94, 051108 (2009).

${ }^{6}$ C. Kadow, S. B. Fleischer, J. B. Ibbetson, J. E. Bowers, A. C. Gossard, J. W. Dong, and C. J. Palmstrom, Appl. Phys. Lett. 75, 3548 (1999).

${ }^{7}$ M. Griebel, J. H. Smet, D. C. Driscoll, J. Kuhl, C. A. Diez, N. Freytag, C. Kadow, A. C. Gossard, and K. V. Klitzing, Nat. Mater. 2, 122 (2003).

${ }^{8}$ G. M. Loubriel, F. J. Zutavern, A. G. Baca, H. P. Hjalmarson, T. A. Plut, W. D. Helgeson, M. W. O’Malley, M. H. Ruebush, and D. J. Brown, IEEE Trans. Plasma Sci. 25, 124 (1997).

${ }^{9}$ C. Kadow, A. W. Jackson, A. C. Gossard, S. Matsuura, and G. A. Blake, Appl. Phys. Lett. 76, 3510 (2000).

${ }^{10}$ J. J. Liou and J.-S. Yuan, IEE Proc. G 139, 350 (1992).

${ }^{11}$ L. Lin, Y. Yue-Hui, and P. Ya-Bin, Chin. Phys. B 17, 2627 (2008).

${ }^{12}$ R. P. Joshi, P. Kayasit, N. Islam, E. Schamiloglu, C. B. Fledderman, and J. Schoenberg, J. Appl. Phys. 86, 3833 (1999).

${ }^{13}$ P. Kayasit, R. P. Joshi, N. E. Islam, E. Schamiloglu, and J. Gaudet, J. Appl. Phys. 89, 1411 (2001).

${ }^{14}$ R. Gunda, D. S. Gleason, K. Kelkar, P. Kirawanich, W. C. Nunnally, and N. E. Islam, IEEE Trans. Plasma Sci. 34, 1697 (2006).

${ }^{15}$ B. Vermeersch, G. Pernot, H. Lu, J.-H. Bahk, A. Shakouri, and A. Gossard, 14th International Conference on Phonon Scattering in Condensed Matter, Phonons 2012, Ann Arbor, MI (AIP, New York, 2012), pp. 29-30, http://www.umich.edu/ pipe/ Phonons_2012_Abstract_Book.pdf.

${ }^{16}$ Y. Ezzahri and A. Shakouri, Phys. Rev. B 79, 184303 (2009).

${ }^{17}$ T. Q. Qiu and C. L. Tien, Int. J. Heat Mass Transfer 37, 2789 (1994).
${ }^{18}$ K.-C. Tseng and J.-R. Tsai, Numer. Heat Transfer, Part A 53, 726 (2007).

${ }^{19}$ L. Guo, S. L. Hodson, T. S. Fisher, and X. Xu, J. Heat Transfer 134, 042402 (2012)

${ }^{20}$ T. Q. Qiu, T. Juhasz, C. Suarez, W. E. Bron, and C. L. Tien, Int. J. Heat Mass Transfer 37, 2799 (1994).

${ }^{21}$ C. Guo and A. J. Taylor, Int. J. Mod. Phys. B 15, 101 (2001).

${ }^{22}$ A. Melnikov, I. Razdolski, T. O. Wehling, E. T. Papaioannou, V. Roddatis, P. Fumagalli, O. Aktsipetrov, A. I. Lichtenstein, and U. Bovensiepen, Phys. Rev. Lett. 107, 076601 (2011).

${ }^{23}$ E. R. Brown, Appl. Phys. Lett. 75, 769 (1999).

${ }^{24}$ J. E. Bjarnason, T. L. J. Chan, A. W. M. Lee, E. R. Brown, D. C. Driscoll, M. Hanson, A. C. Gossard, and R. E. Muller, Appl. Phys. Lett. 85, 3983 (2004).

${ }^{25}$ T. H. Lee, Planar Microwave Engineering: A Practical Guide to Theory, Measurement, and Circuits (Cambridge University Press, New York, 2004) .

${ }^{26}$ Ioffe Physical Technical Institute, http://www.ioffe.ru/SVA/NSM/ Semicond/GaAs/electric.html

${ }^{27}$ D. G. Cahill, Rev. Sci. Instrum. 75, 5119 (2004).

${ }^{28}$ D. G. Cahill, W. K. Ford, K. E. Goodson, G. D. Mahan, A. Majumdar, H. J. Maris, R. Merlin, and S. R. Phillpot, J. Appl. Phys. 93, 793 (2003).

${ }^{29}$ B. C. Gundrum, D. G. Cahill, and R. S. Averback, Phys. Rev. B 72, 245426 (2005).

${ }^{30}$ G. Tessier, S. Holé, and D. Fournier, Appl. Phys. Lett. 78, 2267 (2001).

${ }^{31}$ V. Pasiskevicius, A. Deringas, and A. Krotkus, Appl. Phys. Lett. 63, 2237 (1993).

${ }^{32}$ A. E. Iverson and D. L. Smith, IEEE Trans. Electron Devices 34, 2098 (1987).

${ }^{33}$ A. J. Schmidt, X. Chen, and G. Chen, Rev. Sci. Instrum. 79, 114902 (2008).

${ }^{34}$ L. Prechtel, M. Padilla, N. Erhard, H. Karl, G. Abstreiter, A. Fontcuberta, I. Morral, and A. W. Holleitner, Nano Lett. 12, 2337 (2012).

${ }^{35}$ T. Motet, J. Nees, S. Williamson, and G. Mourou, Appl. Phys. Lett. 59, 1455 (1991). 